\title{
Hybridization of the Ant Colony Optimization with the K-Means Algorithm for Clustering
}

\author{
Sara Saatchi and Chih Cheng Hung \\ Department of Computer Science, \\ Southern Polytechnic State University, \\ 1100 South Marietta Parkway, Marietta, GA 30060, USA \\ \{ssaatchi, chung\} @spsu.edu
}

\begin{abstract}
In this paper the novel concept of ACO and its learning mechanism is integrated with the K-means algorithm to solve image clustering problems. The learning mechanism of the proposed algorithm is obtained by using the defined parameter called pheromone, by which undesired solutions of the K-means algorithm is omitted. The proposed method improves the K-means algorithm by making it less dependent on the initial parameters such as randomly chosen initial cluster centers, hence more stable.
\end{abstract}

\section{Introduction}

Image segmentation plays an essential role in interpretation of the various kinds of images. Image segmentation techniques can be grouped into several categories such as Edge-based segmentation, Region-oriented segmentation, Histogram-thresholding, and clustering algorithms [1]. The aim of clustering algorithm is to aggregate data pixels into groups or clusters such that pixels in each group are similar to each other and different from other groups. There are various techniques for clustering including the K-means algorithm which is based on the similarity between pixels and the specified cluster centers. The behavior of the K-means algorithm mostly is influenced by the number of clusters specified and the random choice of initial cluster centers. In this study we concentrate on the latter, where the results are less dependent on the initial cluster centers chosen, hence more stabilized, by introducing a hybrid technique using the K-means and the Ant Colony Optimization (ACO) heuristic.

The ACO algorithm was first introduced and fully implemented in [2] on the traveling salesman problem (TSP) which can be stated as finding the shortest closed path in a given set of nodes that passes each node once. The ACO algorithm is one of the two main types of swarm intelligent techniques. Swarm intelligence is inspired from the collaborative behavior of social animals such as birds, fish and ants and their amazing formation of flocks, swarms and colonies. By observing and simulating the interaction of these social animals, a variety of optimization problems are solved. The other type of swarm intelligent techniques is the particle swarm optimization (PSO) algorithm. The algorithm consists of a swarm of particles flying through the search space [3]. Parameters of position and velocity are used to represent the potential solution to the problem and the dynamic of the swarm respectively. The velocity of 
each particle is modified according to its experience and that of its neighbors which changes the position of the particle in order to satisfy the objective. The ACO algorithm which we are focused on, is based on a sequence of local moves with a probabilistic decision based on a parameter, called pheromone as a guide to the objective solution. There are algorithms that while they follow the cited procedure of ACO algorithm, they do not necessarily follow all the aspects of it, which we informally refer to as ant-based algorithm or simply ant algorithm.

There are several ant-based approaches to clustering which are based on the stochastic behavior of ants in piling up objects. In most of the approaches [4-8], the ant algorithm is applied to numeric data which are treated as objects. Ants pick up an object and drop it on a heap where there is the most similarity according to a probability. In $[4,5,6]$ the ant based algorithm is applied initially to form the initial set of clusters, and then the K-means algorithm is used to remove classification error and to assign objects that are left alone, to a cluster. The ant based algorithm is applied again on heaps of objects rather than single object and then the K-means algorithm is used for the same reason as before. The algorithm resolves the problem of local minimal by the randomness of ants. In [4] the idea of density is introduced to determine where the heaps and scattered objects are to increase the searching speed and efficiency of the algorithm. Although in $[4,5,6]$ the K-means algorithm was used, only the stochastic movement of the ants are considered, not the learning mechanism of the ACO through the so called pheromone. In [8] a similar approach is used except that after the ant based initialization, Fuzzy C-Means (FCM) algorithm is used to refine these clusters. In [9] the ACO algorithm is combined with genetic algorithms and simplex algorithms to build a learning model for optimal mask, which is used to discriminate texture objects on aerial images. The ACO has been applied to various problems. An ACO heuristic was introduced for solving maximum independent set problems in [10] where uses the pheromone concept to obtain a learning mechanism.

In this study the ACO algorithm is implemented and it is integrated with the Kmeans algorithm to solve clustering problems and it is applied to images. For clustering as the goal, $m$ number of ants is chosen for clustering. Solutions to the problem will be generated by the ants independently. The best solution will be chosen from these solutions and the assigned pheromone to this solution is incremented. The next $m$ ants are inspired by the previous $m$ ants and start making their own solution. This is repeated until a certain number of iterations when the optimal solution is achieved. Decision based on the pheromone amount may be able to eliminate the undesired solutions which are probable to occur, making the algorithm more stable and the solutions more desirable. The advantage of this algorithm over the K-means is that the influence of the improperly chosen initial cluster centers will be diminished after the best solution is chosen and marked with the pheromone over a number of iterations. Therefore it will be less dependent on the initial parameters such as randomly chosen initial cluster centers and more stabilized while it is more likely to find the global solution rather than the local.

In Section 2 the K-means algorithm is reviewed briefly. Section 3 describes the ACO algorithm. In Section 4 the proposed hybrid ACO-K-means clustering algorithm is discussed. Experimental results are presented in Section 4 and the conclusion and future work are discussed in Section 5. 


\section{The K-Means Algorithm}

The K-means algorithm, first introduced in [11] is an unsupervised clustering algorithm which partitions a set of object into a certain number of clusters. The Kmeans algorithm is based on the minimization of a performance index which is defined as the sum of the squared distances from all points in a cluster domain to the cluster center [12]. First K random initial cluster centers are chosen. Then each sample is assigned to a cluster based on the minimum distance to the cluster centers. Finally cluster centers are updated by calculating the average of the values in each cluster and this will be repeated until cluster centers no longer change.

The K-means algorithm tends to find the local minima rather that the global therefore it is heavily influenced by the choice of initial cluster centers and the distribution of data. Most of the time the results become more acceptable when initial cluster centers are chosen relatively far apart since the main clusters in a given data are usually distinguished in such a way. If the main clusters in a given data are close in characteristics the K-means algorithm fails to recognize them when it is left unsupervised. For its improvement the K-means algorithm needs to be associated with some optimization procedures in order to be less dependent on a given data and initialization.

\section{The ACO Algorithm}

The ACO heuristic has been inspired by the observation on real ant colony's foraging behavior and on that ants can often find the shortest path between food source and their nest [10]. This is achieved by a deposited and accumulated chemical substance called pheromone by the passing ant which goes towards the food. In its searching the ant uses its own knowledge of where the smell of the food comes from (we call it as heuristic information) and the other ants' decision of the path toward the food (pheromone information). After it decides its own path, it will confirm the path by depositing its own pheromone making the pheromone trail denser and more probable to be chosen by other ants. This is a learning mechanism ants follow beside their own recognition of the path. As a result of this consulting of ants with the ants already done a job, the best path which is the shortest will be marked from the nest towards the food.

ACO uses this learning mechanism. Furthermore, in the ACO algorithm, the pheromone level is updated based on the best solution obtained by a number of ants and the pheromone amount that is deposited by the succeeded ant is defined to be proportional to the quality of the solution it produces. For the real ants, the best solution is the shortest path and it will be marked with a strong pheromone trail. In the short path problem using ACO algorithm, the pheromone amount deposited is inversely proportional to the length of the path. For a given problem the pheromone can be set to be proportional to any criteria of the desired solution. In the clustering method we will introduce below, the criteria includes the similarity of data in each cluster, distinction of the clusters and compactness of them. More details on the ACO algorithm can be found in [2]. 


\section{The Proposed Hybrid ACO-K-Means Clustering Algorithm}

Our approach to the problem is very similar to the K-means strategy. It starts by choosing the number of clusters and a random initial cluster center for each cluster. ACO plays its part in assigning each pixel to a cluster. This is done according to a probability which is inversely dependent to the distance (similarity) between the pixel and cluster centers and a variable, $\tau$, representing the pheromone level. Pheromone is defined to be dependent to minimum distance between each pair of cluster centers and inversely dependent on the distances between each pixel and its cluster center. So the pheromone gets bigger when cluster centers get far apart and clusters tend to be more compact (our criterion for best solution), making the probability of assigning a pixel to that cluster high. Pheromone evaporation is considered to weaken the influence of the previously chosen solutions, which are less likely to be desired. Similar to the Kmeans algorithm, at this stage new cluster centers are updated by calculating the average of the pixels in each cluster and this will be repeated until cluster centers no longer change. But unlike K-means, this algorithm doesn't stop here. It is assumed that the described clustering job is performed by an ant, and there are $m$ ants repeating this job, each with their own random initialization and they all will end up with a solution. A criterion is defined to find the best solution and the pheromone level is updated accordingly for the next set of $m$ ants as a leading guide. A termination criterion will stop the algorithm and an optimal solution is resulted.

The algorithm starts by assigning a pheromone level $\tau$ and a heuristic information $\eta$ to each pixel. Then each ant will assign each pixel to a cluster with the probability $P$. This assignment of parameters is shown in Table 1 , where $P$ is obtained from Eq. 1 [2]:

$$
P_{(i, X n)}=\frac{\tau_{(i, X n)}^{\alpha} \eta_{(i, X n)}^{\beta}}{\sum_{i=0}^{K} \tau_{(i, X n)}^{\alpha} \eta_{(i, X n)}^{\beta}}
$$

where $P_{(i, X n)}$ is the probability of choosing pixel $X_{n}$ in cluster $i, \tau_{(i, X n)}$ and $\eta_{(i, X n)}$ are the pheromone and heuristic information assigned to pixel $X_{n}$ in cluster $i$ respectively, $\alpha$ and $\beta$ are constant parameters that determines the relative influence of the pheromone and heuristic information, and $\mathrm{K}$ is the number of clusters. Heuristic information $\eta_{(i, X n)}$ is obtained from:

$$
\eta_{(i, X n)}=\frac{\kappa}{\operatorname{ColDist}\left(X_{n}, C_{i}\right) * \operatorname{Phys} \operatorname{Dist}\left(X_{n}, C_{i}\right)}
$$

where $X_{n}$ is the $n^{\text {th }}$ pixel and $C_{i}$ is the $i^{\text {th }}$ cluster center. $\operatorname{ColDist}\left(X_{n}, C_{i}\right)$ is the color distance between $X_{n}$ and $C_{i}$, and PhysDist $\left(X_{n}, C_{i}\right)$ is the physical (geometrical) distance between $X_{n}$ and $C_{i}$. Constant $\kappa$ is used to balance the value of $\eta$ with $\tau$.

The value for the pheromone level $\tau$ assigned to each pixel is initialized to 1 so that it doesn't have effect on the probability at the beginning. This pheromone should become bigger for the best solution we are looking for. 
Table 1. Assignment of parameters to each pixel in each clustering solution obtained by an ant

\begin{tabular}{|c|c|c|c|c|}
\hline Cluster\#\Pix & $\mathrm{X}_{0}$ & $\mathrm{X}_{1}$ & $\ldots$ & $\mathrm{Xn}$ \\
\hline 0 & $\mathrm{P}_{(0, \mathrm{X} 0)}$ & $\mathrm{P}_{(0, \mathrm{X} 1)}$ & & $\mathrm{P}_{(0, \mathrm{Xn})}$ \\
\hline 1 & $\mathrm{P}_{(1, \mathrm{X} 0)}$ & $\mathrm{P}_{(1, \mathrm{X} 1)}$ & & $\mathrm{P}_{(1, \mathrm{Xn})}$ \\
\hline 2 & $\mathrm{P}_{(2, \mathrm{X} 0)}$ & $\mathrm{P}_{(2, \mathrm{X} 1)}$ & & $\mathrm{P}_{(2, \mathrm{Xn})}$ \\
\hline$\ldots$ & & & & \\
\hline
\end{tabular}

Suppose $m$ number of ants is chosen for clustering on an image. Each ant is giving its own clustering solution. After $m$ ants have done their clustering, the current best solution is chosen and the assigned pheromone to this solution is incremented. Also cluster centers are updated by the cluster centers of the current best solution. The next $m$ ants inspire from the previous $m$ ants and start making their own solution. In each of iterations, each one of the $m$ ants finds its solution based on the best solution found by the previous $m$ ants, and the best solution is found for the next $m$ ants. This is repeated until a certain amount of times where the overall best solution is achieved.

The best solution in each of iterations is chosen according to two factors; distance between cluster centers and sum of the color and physical distances between each pixel and its cluster center (similarity and compactness of clusters). For the best solution to choose: 1) Distance between cluster centers should be large so the clusters are further apart, 2) The sum of the color distances between each pixel and its cluster center should be small so that each cluster becomes more similar in color and 3) The sum of the distances between each pixel and its cluster center should be small so that each cluster becomes more compact. To achieve the first one, for each clustering performed by ant $k(k=1, \ldots, m)$, we compute the distances between every pair of cluster centers and sort these distances then we pick the minimum distance $\operatorname{Min}(k)$. Now we compare all these minimums performed by all the ants, and pick the maximum of them $\left[\operatorname{Min}\left(k^{\prime}\right)\right]$. To achieve the second and third, for each clustering performed by ant $\mathrm{k}$ we compute the sum of the distances between each pixel and its cluster center, and sort these sum of the distances. Then we pick the maximum and compare all these maximums performed by all ants, and pick the minimum of them. The second maximum and third maximum of the solutions are compared in the same way and the minimum is picked. Since every ant has its own solution, solutions are being voted based on their advantages and the solution with the larger vote is selected as the best solution.

After the best solution is found, the pheromone value is updated according to Eq. 3 [10]:

$$
\tau_{(i, X n)} \leftarrow(1-\rho) \tau_{(i, X n)}+\Sigma_{i} \Delta \tau_{(i, X n)} .
$$

Where $\rho$ is the evaporation factor $(0 \leq \rho<1)$ which causes the earlier pheromones vanish over the iterations. Therefore as the solution becomes better, the corresponding pheromone have more effect on the next solution rather than the earlier pheromones which correspond to the initial undesired solutions found. $\Delta \tau_{(i, X n)}$ in Eq. 3 is the amount of pheromone added to previous pheromone by the succeeded ant, which is obtained from: 


$$
\Delta \tau_{(i, X n)}= \begin{cases}\frac{Q^{*} \operatorname{Min}\left(k^{\prime}\right)}{A v g \operatorname{ColDist}\left(k^{\prime}, i\right) * \operatorname{AvgPhys} \operatorname{Dist}\left(k^{\prime}, i\right)} & \text { if } \mathrm{X}_{\mathrm{n}} \text { is a member of cluster i. } \\ 0 & \text { otherwise. }\end{cases}
$$

In Eq. 4, $Q$ is a positive constant which is related to the quantity of the added pheromone by ants, $\operatorname{Min}\left(k^{\prime}\right)$ is the maximum of the minimum distance between every two cluster centers obtained by ant $k^{\prime}, A v g \operatorname{ColDist}\left(k^{\prime}, i\right)$ is the average sum of the color distances and AvgPhysDist $\left(k^{\prime}, i\right)$ is the average sum of the physical distances, between each pixel and its cluster center $i$ obtained by ant $k^{\prime}$. Min $\left(k^{\prime}\right)$ causes the pheromone become bigger when clusters get more apart and hence raise the probability. Avg ColDist $\left(k^{\prime}, i\right)$ and AvgPhysDist $\left(k^{\prime}, i\right)$ cause the pheromone become bigger when the cluster has more similar pixels and is more compact respectively. In other words the more the $\operatorname{Min}\left(k^{\prime}\right)$ the more apart our clusters are which is desired and the bigger pheromone, and the less the AvgColDist $\left(k^{\prime}, i\right)$ and AvgPhysDist $\left(k^{\prime}, i\right)$, the more similar and compact our clusters are which is desired and the bigger the pheromone.

Next, cluster centers are updated by the cluster centers of the best solution. This is repeated until a certain amount of times where the best of the best solution is achieved. The algorithm is described below:

Step 1: Initialize pheromone level to 1 , and the number of clusters to $K$ and number of ants to $m$.

Step 2: Initialize $m$ sets of $K$ different random cluster centers to be used by $m$ ants.

Step 3: For each ant, let each pixel $x$ belong to one cluster with the probability given in Eq. 1.

Step 4: Calculate new cluster center; If the new cluster centers converge to the old ones, go to next step otherwise, go to Step 3 .

Step 5: Save the best solution among the m solutions found.

Step 6: Update the pheromone level on all pixels according to the best solution.

Step 7: Update cluster centers by the cluster center values of the best solution.

Step 8:If the termination criterion is satisfied go to next step otherwise, go to Step3.

Step 9: Output the optimal solution.

\section{Simulation Results}

In the simulation the parameters involved are set as follows. Parameters $\alpha, \beta$ and $\kappa$ are used to keep the values of $\tau$ and $\eta$ in the same order. The values set was $\alpha=2, \beta=5$, and $\kappa=1000$. The parameters $Q$ controls the added amount of pheromone and $\rho$ eliminates the influence of the earlier added pheromone and they are set to be, $Q=10$ and $\rho=0.8$. The number of ants is chosen to be $m=10$.

The results for both the K-means and Hybrid ACO-K-means are compared in Figs. 1 and 2 on two different images. In both cases the number of clusters to be found is 3 . Different runs of the proposed algorithm are shown in Figs. 1 (d) and (e), which compared to the ground truth data shown in Fig. 1 (b) show a better result with respect to the K-means. Figs. 2 (c) and (d) are the results for another image. As it can 
be seen there are some scattered dots over the segmented images. In the first look this can be thought of the instability of the algorithm, but contradiction of this is proved in Figs. 3 and 4 which we will discuss later. These dotted regions do not influence the big picture of the clustering and it is due to the randomness of the algorithm.

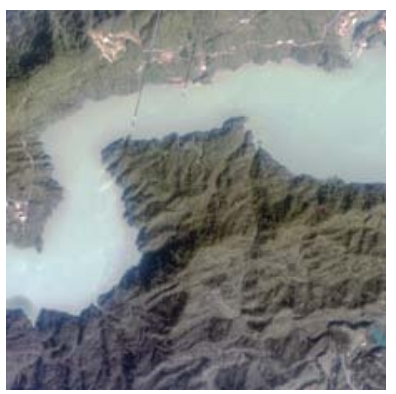

(a)

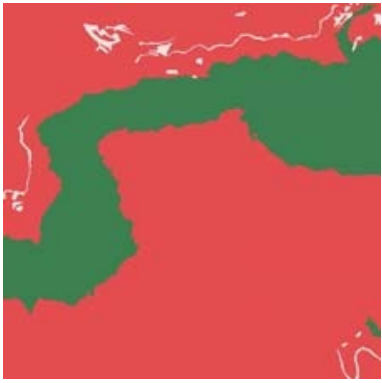

(b)

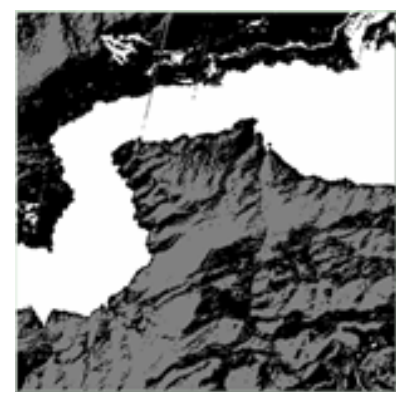

(c)

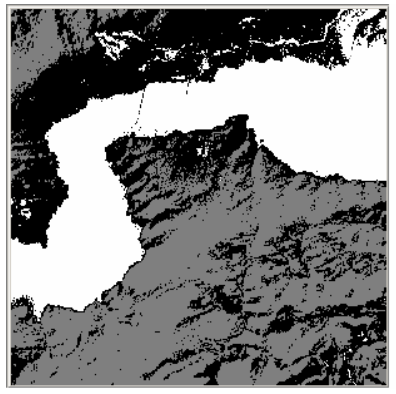

(d)

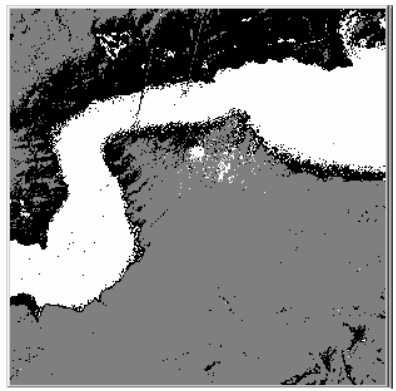

(e)

Fig. 1. a) An original image, b) The ground truth data, c) K-means segmentation results, d \& e) Hybrid ACO-K-means results with $K=3$ and different runs

In Fig. 3 improper seeds are forced to show the case in which initial seed values are not properly chosen. Fig. 3 (a) and (b) are the only results of the K-means in this case which shows some information loss, where Figs. 3 (c) and (d) show the results of ACO-K-means which shows that the information is kept. This shows that even when improper seeds are forced to the Hybrid ACO-K-means, since there are several solutions to compete, eventually the improper solution will be omitted and the proper one wins as the answer. This indicates the stability of the Hybrid ACO-K-means algorithm. The stability of the proposed algorithm is further confirmed in Fig. 4 where different runs of both K-means and ACO-K-means are shown. There are some runs of the K-means algorithm which lose the information Fig. 4 (c), due to improper initialization which it is not the case in ACO-K-means. 


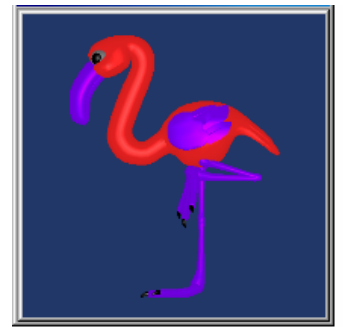

(a)

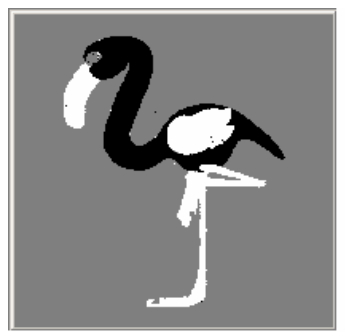

(c)

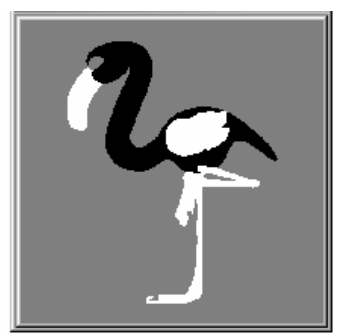

(b)

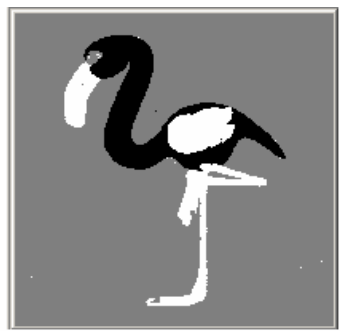

(d)

Fig. 2. a) An original image, b) K-means results with $K=3$, c \& d) Hybrid ACO-K-means results with $K=3$ and different runs

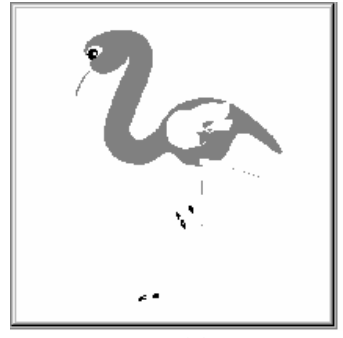

(a)

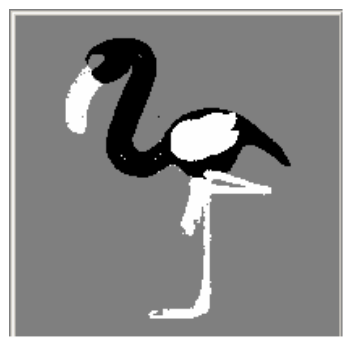

(c)

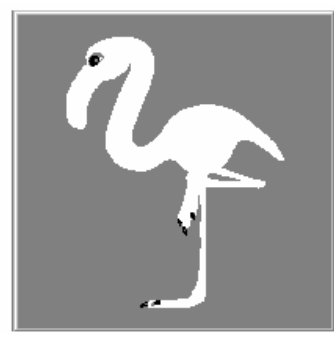

(b)

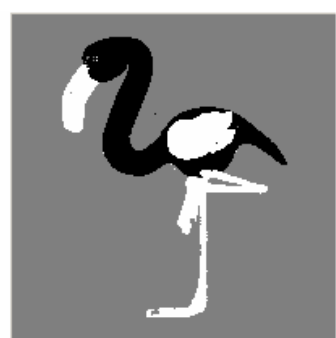

(d)

Fig. 3. a \& b) K-means results with $K=3$ and different runs, where the initial seed values are not properly chosen, c \& d) Hybrid ACO-K-means results with $K=3$ and different runs, where the initial seed values are not properly chosen 


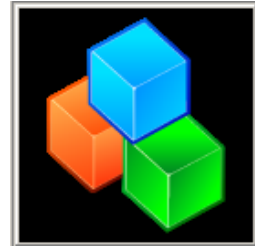

(a)

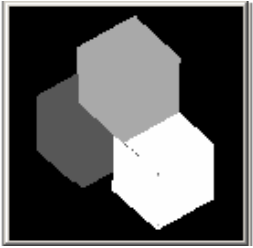

(b)

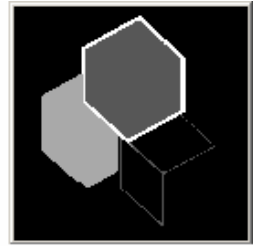

(c)

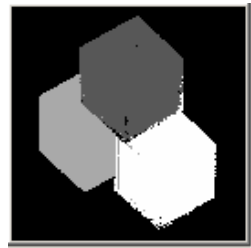

(d)

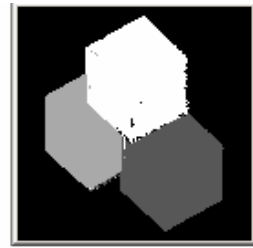

(e)

Fig. 4. a) An original image, b \& c) K-means results with $K=4$ and different runs, d \& e) Hybrid ACO-K-means results with $K=4$ and different runs

\section{Conclusion and Future Work}

In this paper a Hybrid ACO-K-means algorithm is proposed. In this algorithm ants are used to obtain solutions based on their own knowledge and the best solution found by previous ants. The advantage of this algorithm over the K-means algorithm is revealed when initial seeds are chosen improperly. Since a solution is obtained for a number of iterations, most of the possible solutions are available to choose from as the best solution. Therefore over a number of iterations, the influence of the improperly chosen initial cluster centers will be diminished. This best solution is marked with the pheromone and eventually becomes denser causing the best result to become as the final result. Therefore this algorithm is less dependent on the initial parameters such as randomly chosen initial seeds hence more stabilized and it is more likely to find the global solution rather than the local.

The proposed ACO-K-means algorithm is not limited to a particular problem. For a given problem the main problem is to define the heuristic information, the pheromone level and the criteria for the best solution. Therefore with this learning mechanism the problem of finding the desired number of clusters with unsupervised information can also be solved by defining the heuristic and pheromone information and desired criterion for the best solution, as an extension of this work.

\section{Acknowledgments}

The authors would like to thank Arash Karbaschi and Sara Arasteh for valuable discussions and helps. 


\section{References}

1. Gonzalez, R.C., Woods, R.E., Digital Image Processing, Addison-Wesley (1992)

2. Dorigo, M., Maniezzo, V., Colorni, A., Ant system: optimization by a colony of cooperating agents, In: IEEE Transactions on Systems, Man and Cybernetics, Part B, Vol. 26, (1996) 29-41

3. Kaewkamnerdpong, B., Bentley, P.J., Perceptive Particle Swarm Optimization,

4. http://www.cs.ucl.ac.uk/staff/B.Kaewkamnerdpong/bkpb-icannga05.pdf

5. Yuqing, P., Xiangdan, H., Shang, L., The K-means clustering Algorithm Based on Density and Ant Colony, In: Proceedings of the International Conference on Neural Networks and Signal Processing, Vol. 1, Nanjing, China, (2003) 457-460

6. Monmarch'e, N., On data clustering with artificial ants, In: AAAI-99 and GECCO-99 Workshop on Data Mining with Evolutionary Algorithms: Research Directions, A.A. Freitas, ed., Orlando, Florida, (1999) 23-26

7. Monmarch'e, N., Slimane, M., Venturini, G., AntClass: discovery of clusters in numeric data by an hybridization of ant colony with k-means algorithm, Internal Report no. 213 Laboratoire d'Informatique, E3i, Universite de Tours, Tours, France, (1999)

8. Bin, W., Yi, Z., Shaohui, L., Zhongzhi, S., CSIM: A Document Clustering Algorithm Based on Swarm Intelligence, In: Proceedings of the Congress on Evolutionary Computation, Vol. 1, Honolulu, HI, (2002) 477-482

9. Kanade, P.M., Hall, L.O., Fuzzy Ants as a Clustering Concept, In: Proceedings of 22nd International Conference of the North American Fuzzy Information Processing Society, Chicago, IL, (2003) 227-232

10. Zheng, H., Zheng, Z., Xiang, Y., The application of ant colony system to image textute classification [textute read texture], In: Proceedings of the 2nd International Conference on Machine Learning and Cybernetics, Vol. 3, Xi'an, China, (2003) 1491-1495

11. Li, Y., Xu, Z., An Ant Colony Optimization Heuristic for Solving Maximum Independent Set Problems, In: Proceedings of the 5th International Conference on Computational Intelligence and Multimedia Applications, Xi'an, China, (2003) 206-211

12. MacQueen, J.B., Some Methods For Classification and Analysis of Multivariate Observations. In L. M. LeCam and J. Neyman, editors, Proceedings of the Fifth Berkeley Symposium on Mathematical Statistic and Probability, University of California Press, Berkley, CA, (1967) 281-297

13. Tou, J.T., Gonzalez, R.C., Pattern Recognition Principles, Addison-Wesley, (1974) 